\title{
Movement Fluidity Analysis Based on Performance and Perception
}

$\begin{array}{ll}\begin{array}{l}\text { Stefano Piana } \\ \text { University of Genoa } \\ \text { DIBRIS } \\ \text { Genoa, IT } \\ \text { stefano.piana@dist.unige.it }\end{array} & \begin{array}{l}\text { Paolo Alborno } \\ \text { University of Genoa } \\ \text { DIBRIS } \\ \text { Genoa, IT } \\ \text { paoloalborno@gmail.com }\end{array} \\ \begin{array}{l}\text { Radoslaw Niewiadomski } \\ \text { University of Genoa }\end{array} & \begin{array}{l}\text { Maurizio Mancini } \\ \text { University of Genoa }\end{array} \\ \begin{array}{l}\text { DIBRIS } \\ \text { Genoa, IT }\end{array} & \begin{array}{l}\text { DIBRIS } \\ \text { radek@infomus. IT }\end{array} \\ & \text { maurizio.mancini@unige.it } \\ \text { Gualtiero Volpe } & \\ \text { University of Genoa } & \text { Antonio Camurri } \\ \text { DIBRIS } & \text { University of Genoa } \\ \text { Genoa, IT } & \text { DIBRIS } \\ \text { gualtiero.volpe@gmail.com } & \text { Genoa, IT } \\ & \text { antoniocamurri@gmail.com }\end{array}$

Permission to make digital or hard copies of part or all of this work for personal or classroom use is granted without fee provided that copies are not made or distributed for profit or commercial advantage and that copies bear this notice and the full citation on the first page. Copyrights for third-party components of this work must be honored. For all other uses, contact the owner/author(s). Copyright is held by the

author/owner(s)

CHI'16 Extended Abstracts, May 7-12, 2016, San Jose, CA, USA.

ACM 978-1-4503-4082-3/16/05

http://dx.doi.org/10.1145/2851581.2892478

\begin{abstract}
In this work we present a framework and an experimental approach to investigate human body movement qualities (i.e., the expressive components of non-verbal communication) in $\mathrm{HCl}$. We first define a candidate movement quality conceptually, with the involvement of experts in the field (e.g., dancers, choreographers). Next, we collect a dataset of performances and we evaluate the perception of the chosen quality. Finally, we propose a computational model to detect the presence of the quality in a movement segment and we compare the outcomes of the model with the evaluation results. In the proposed on-going work, we apply this approach to a specific quality of movement: Fluidity. The proposed methods and models may have several applications, e.g., in emotion detection from full-body movement, interactive training of motor skills, rehabilitation.
\end{abstract}

\section{Author Keywords}

movement; analysis; Fluidity; perception; evaluation; performance; dance.

\section{ACM Classification Keywords}

H.5.2 [User Interfaces]; H.1.2 [User/Machine Systems]; J.5 [Arts and Humanities] 


\section{Introduction}

Human-human interaction involves verbal (e.g., speech) and non-verbal (e.g., voice prosody, facial expressions, gestures, full-body movements in general, and so on) communication signals. The meaning of non-verbal signals is determined by two components: their "shape" and "expressive quality". The role of the former has been widely studied [15], while the latter, i.e., "how a particular mental intention is communicated through movement expressive quality" [18], has been addressed only recently. For example, researchers investigated human movement in order to identify the expressive qualities that communicate emotional content $[23,1,20]$. Other studies exploited music and dance performances as a test-bed to automatically analyze the communication of social roles in groups of people performing collaborative tasks [9].

One of the goals of multimodal interfaces is to transfer the human-human communication paradigm to HumanComputer Interaction. In the last years, a wide number of studies addressed the automated detection of user's fullbody movement qualities, with the long-term goal of endowing machines with the capability to "decode" human's non-verbal behavior and signals. Our on-going research is part of the more general scenario of modeling human body movement quality, and in particular its expressive components in non-verbal communication. We are mainly focused on how movement qualities are perceived by an external observer. The importance of this challenging scenario is evident in several domains and applications, such as diagnostic aspects of psychopathological disorders [23], therapy and rehabilitation [21], expressive and natural interfaces [3], and affective computing $[2,8]$

Compared to previous work on expressive qualities analysis, our approach takes into account human perception of a professional performance. We first define the target quality conceptually, with the contribute of experts. Next, we collect a dataset of performances by professional dancers. Then, we evaluate the perception of the target quality in their performances. Finally, we propose a computational model allowing us to detect the presence of the quality in a movement segment and we compare the outcomes of the model with the evaluation results. In our work, which is still in-progress, we apply this approach to a specific quality of movement: Fluidity.

\section{Multi-layer Framework}

Qualities of movement can be conceived in a multi-layer framework [3]: a first physical layer concerns kinematics, e.g., trajectories and velocities of joints, or the silhouette of the body. Biomechanic features of single joints at a small time scale (few milliseconds) are defined at a second (higher) feature layer: for example, "smoothness", as defined in the literature in terms of minimum jerk $[22,16]$ or in terms of curvature of velocity trajectories [12]. Mazzarino et al. [13] performed another study on a similar biomechanical feature named "fluency", exploiting the variation of quantity of motion to characterize it [3]. A third (even higher) layer addresses more complex qualities, usually referred to groups of joints or to the whole body, and requires significant temporal intervals to observe (e.g., rhythmic properties typically require a range of $0.5 s-5 s$; [6]). This layer is typical of the qualities of Laban's Effort [11]: Flow, Weight, Time, Space.

\section{Fluidity of Movement: Definition}

In this paper we focus on an important movement quality belonging to the third layer of the above framework: Fluidity.

Fluidity is often considered as a synonym of "good" movement (e.g., in certain dance styles), and is much more than 


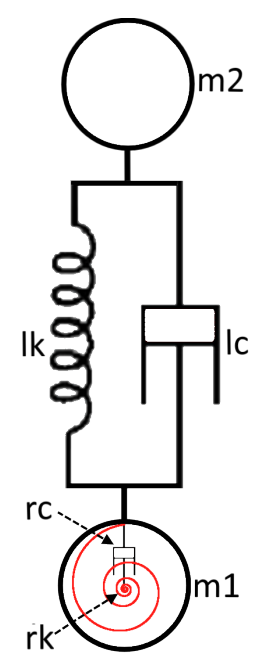

Figure 1: a simple model, two masses $(m 1$ and $m 2)$ are linked by a spring $(l k)$, and the resulting body segment is influenced by a rotational spring $(r k)$ that controls its rotation and movement. "smoothness", which is referred to the movement of a single joint. Furthermore, Fluidity is one of the properties that seem to contribute significantly to perception of emotions [3]. Fluidity has been investigated by the work of Caridakis et al. [4] on hands trajectories, where it was computed as the sum of the variance of the norms of the hands' motion vectors. Piana et al. [19] studied human motion trajectories and defined a Fluidity index based on the minimum jerk law.

Starting from literature on biomechanics and psychology, and by conducting interviews and movement recordings with experts in human movement such as choreographers and dancers, we propose the following definition of Fluidity of movement:

Definition 1 A Fluid movement can be performed by a part of the body or by the whole body and is characterized by the following properties.

Property 1 (P1): the movement of each involved joint of the (part of) the body is smooth, following the standard definitions in the literature of biomechanics [22, 16, 19].

Property 2 (P2): the energy of movement (energy of mus cles) is free to propagate along the kinematic chains of (parts of) then body (e.g., from head to trunk, from shoulders to arms) according to a coordinated wave-like propagation. That is, there is an efficient propagation of movement along the kinematic chains, with a minimization of dissipation of energy.

These two properties account for a wide range of fluid movements. For example, let us consider the Flow quality (Free/Bound) of Laban's Effort [11]. A Bound movement is performed under full control; a Free movement, once started, cannot be interrupted until its completion (e.g., a jump, a throw). A Fluid movement of a shoulder and its arm, is characterized by a smooth movement of each joint, and it can be Free (a wave-like movement propagating freely from the shoulder to the arm, the forearm, the hand, the fingertips to the outer space, like in a lashing) or Bound (a wave-like movement where the propagation from the shoulder to the fingertips is fully controlled, possibly very slow).

More in general, Fluidity is a quality that in real movements co-exists with other qualities.

This approach, based on our multi-layered conceptual framework, can be applied to a number of other movement qualities, e.g., Impulsivity, Weight (Light/Heavy). This is one of the main research activities in the DANCE EU ICT H2020 Project ${ }^{1}$. In this paper, we focus only on Fluidity, and we explore an implementation of the above definition of Fluidity in terms of a simple physical model based on mass-dampersprings, showing how this implementation explains the evaluations of a population of subjects asked to rate Fluidity from a sample dataset of recordings.

\section{A Human Mass-Spring-Damper Model}

In this work we propose a model for human movement analysis. The model is based on a Mass-Spring-Damper model. Simple Mass-spring models have been used to analyse human movement: in $[24,5,7,17]$ authors created simple mass-spring models to simulate human gait, run, and jump. Authors of [10] generated a set of mass-spring models to simulate different dance verbs. The model we propose represents the human body as a set of interconnected masses, each mass (estimated using anthropometric tables [14]) represents a body's joint. The model contains two kinds of spring: we define the first type of spring as longitudinal springs $(l k)$, joints are connected together by this kind of spring, we define two masses connected by a longitudinal springs ( $r k$ ) as a body segment. We define the second type

\footnotetext{
${ }^{1}$ H2020 ICT Project n.645553 http://dance.dibris.unige.it/
} 


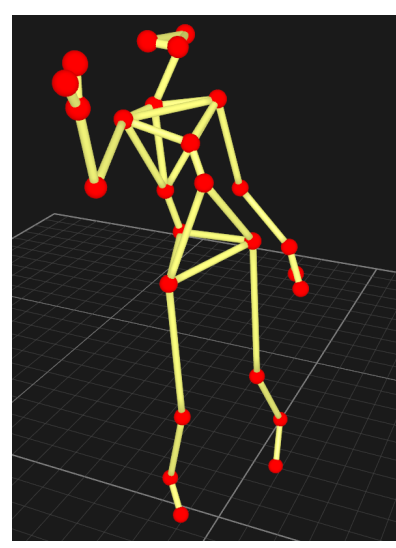

Figure 2: a frame of stick-figure animation. of spring as rotational springs, that impress rotational forces on body segments; Figure 1 represents an example of the model with a single body segment. The proposed model can be used to analyse, filter, synthesize and/or alter movements. The response of the model to the same stimuli can vary tuning its parameters (i.e., spring stiffness, masses of the joints, damping coefficients) allowing to simulate a very large number of different conditions (i.e., a stiff/rigid body vs a fluid one).

\section{Dataset}

We recorded short performances of professional dancers who were asked to exhibit full body movements with a requested expressive quality: the dancers were given short instructions (scenarios).

Two professional female dancers participated to the recording sessions. At the beginning of each session, the dancers were given our definition of Fluidity. Then, they were instructed to repeat predefined movements (e.g., to pick up the object from the floor, or to throw an object) using the requested expressive qualities (e.g., fluid vs non-fluid). For the recordings, a Qualisys motion capture system was used at $100 \mathrm{~Hz}$, synchronized with video (1280x720,50fps). The resulting data consists of 3D positions of twenty six markers (see Figure 2).

\section{Dataset Evaluation}

Our aim is to define a computational model to detect the presence of Fluidity. Our dataset needs to be validated from the observer's perception point of view and in terms of fluid vs non-fluid qualities.

For this reason we setup an online perceptive study. Participants were asked to watch stick-figure animations of a skeleton (i.e., with no audio and no facial expressions, see
Figure 2). After seeing an animation they had to answer whether the following properties were present in the animation, by using 5 point Likert scale from "I totally disagree" to "I totally agree":

Person's energy of movement (e.g., energy of muscles) is free to flow between body's regions (e.g., from trunk, to arms, from head to trunk to feet, and so on), the same way a wave propagates in a fluid (e.g., when a stone is thrown into a pond, and circular waves radiate from the point where the stone fell down)

It is worth to notice that the proposed text in the evaluation study did not contain the name of the expressive quality the study was focused on (i.e., Fluidity). This choice was made intentionally to avoid participants (in particular those who do not have any experience in dance) to provide their own interpretation of Fluidity.

The evaluation set consisted of 42 stick-figures animations: 21 segments where the dancer was asked to explicitly perform movements characterized by high Fluidity and (ii) 21 segments, where she was expressing non-fluid qualities (e.g., rigidity). Segments duration is between 3 and 22 seconds (total duration $5 \mathrm{~m}$ and $34 \mathrm{~s}$ ).

A web page displayed single full-body skeleton animations of motion capture data corresponding to one segment. Participants could watch each animation as many times as they wanted. Each participant had to evaluate maximum 20 animations. Animations were displayed in a random order: each new animation was chosen among the animations that received the smaller number of evaluations. In this way, we obtained a balanced number of evaluations for all segments. 


\section{Computation Algorithm}

In this work, as a proof of concept, our spring-mass model was used to simulate a dancer's body and to compare the various recorded performances with the movements generated by the model. Since the model was designed (by experimentally tuning its parameters) to generate the smoothest trajectories, it has been used as reference to estimate Fluidity.

In particular, we computed the mean jerk values of the shoulders $(s)$, elbows $(e)$ and hands $(h)$ for both the original measurements and the ones simulated by the model. By measuring the distance of the overall jerk of the captured data and the synthesized one we identified a quantity $J I$ that roughly estimates the Fluidity of movement of a given segment. The $J I$ index is computed at frame $k$ as follows:

$$
J I_{k}=J I_{k}^{l}+J I_{k}^{r}
$$

where $J I_{k}^{l}$ and $J I_{k}^{r}$ are respectively:

$$
J I_{k}^{l}=\left|\left(\dddot{X}_{k}^{l s}+\dddot{X}_{k}^{l e}+\dddot{X}_{k}^{l h}\right)-\left(\dddot{Y}_{k}^{l s}+\dddot{Y}_{k}^{l e}+\dddot{Y}_{k}^{l h}\right)\right|
$$

and:

$$
J I_{k}^{r}=\left|\left(\dddot{X}_{k}^{r s}+\dddot{X}_{k}^{r e}+\dddot{X}_{k}^{r h}\right)-\left(\dddot{Y}_{k}^{r s}+\dddot{Y}_{k}^{r e}+\dddot{Y}_{k}^{r h}\right)\right|
$$

The procedure for evaluating the Fluidity estimation of a segment is explained in Algorithm 1.

\section{Results}

In total we collected 546 answers from 41 participants (15 females, age $=23.5$ (Mean $=30.7, \mathrm{SD}=5.8$ ), 11 Nationalities (63\% Italy, 10\% France)). Each animation was evaluated 13 times. Figure 3 presents average of user answers, $U E_{i}$, of segments $s_{i}$. while A new frame of the segment $s_{i}$ is available do

let $X_{k}$ be a set of coordinates measured at frame $k$; set $X_{k}$ as target position for the model

let the model evolve and get the simulated set $Y_{k}$; evaluate $J I_{k}$ as in Equation 1;

update mean value $J I_{i}$

wait next data frame;

\section{end while}

Algorithm 1: Fluidity estimation from 3D coordinates:

for each frame $k$ of a MoCap segment $i$, an estimation of the jerkness $J I_{k}$ is computed, finally an estimation of the mean jerkness $J I_{i}$ of the segment is calculated

From Figure 3 it can be seen that segments intended to be fluid can be easily separated from the rest of the segments. Indeed, using the threshold $t r=3.65$ we can define 2 sets of segments: high Fluidity segments (HFS), i.e. $H F S=\left\{s_{i}: U E_{i} \geq t r\right\}$, and low Fluidity segments (LFS), i.e. $L F S=\left\{s_{i}: U E_{i}<t r\right\}$. At the same time, $H F S$ contains all segments in which dancers were asked to express high Fluidity. The means of participants' answers $U E_{i}$ are bigger than 3.65 for segments where the dancers were asked to move fluid, and they are lower than 3.65 for all other segments.

Next, we also applied ANOVA with Intention (fluid Vs. other) as independent variable and the average participants' answers $U E_{i}$ as dependent variable (see Table 1). The participants' answers were significantly higher for segments intended to express a high Fluidity $(F(1,41)=215.102, p<$ .001).

Table 1: results of the perceptive evaluation $(U E)$.

\begin{tabular}{c|cc} 
Intention & Fluid & Other \\
\hline$U E$ & $4.2 \pm 0.29$ & $2.05 \pm 0.61$
\end{tabular}




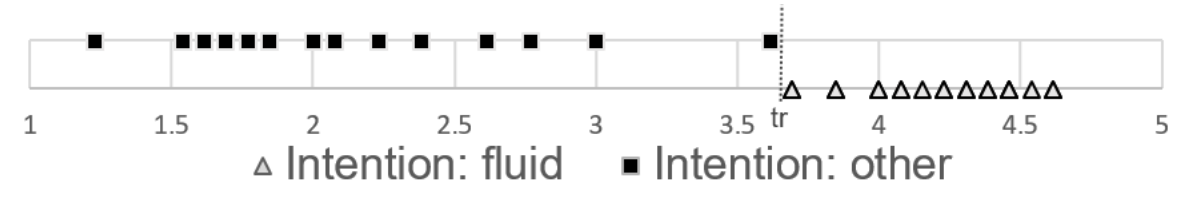

Figure 3: the average scores for 42 segments divided according to the dancers' intention.

Table 2 shows results on the computation of $J I$ on the recorded data, the table shows that movement segments identified as fluid by evaluators are characterized by a statistically significant lower $J I$ index $(F(1,41)=11.45, p<$ .001) than non fluid movements.

Table 2: results of the proposed algorithm.

\begin{tabular}{c|cc} 
Intention & Fluid & Other \\
\hline$J I$ & $0.0198 \pm 0.0004$ & $0.043 \pm 0.0005$
\end{tabular}

The results indicate that the $J I$ index may be useful in identifying fluid movements.

\section{Conclusion}

In this paper we proposed a new definition of full-body movement Fluidity based on the perceptive evaluation of professional dancers' performance. We also proposed an algorithm based on Mass-Spring-Damper model to detect the presence Fluidity in movements. In future we plan to include the data of more dancers. We will also consider a more fine-grained distinction between related but distinctive expressive qualities such as movement lightness, jerkiness or rigidity. Finally we will work on improving our algorithm to compute Fluidity on a continuous scale. Furthermore we will work on the detection of the proposed movement quality in different contexts, we see possible applications in human computer interaction in particular in NUI interfaces, rehabilitation and affective computing, to do so we will work on the extension of the models to be more scalable and work on different devices (i.e., wearables, low cost motion capture devices). Besides Fluidity, we are studying a number of computational models of other movement qualities aiming to translate dance expression from the visual to the auditory channel, by means of interactive sonification techniques.

\section{Acnowledgements}

This research has received funding from the European Union's Horizon 2020 research and innovation programme under grant agreement No 6455533 (DANCE) ${ }^{2}$. DANCE investigates how affective and relational qualities of body movement can be expressed, represented, and analyzed by the auditory channel.

We thank our collegues at Casa Paganini - InfoMus Corrado Canepa, Paolo Coletta, Nicola Ferrari, Simone Ghisio, Ksenia Kolykhalova, and Roberto Sagoleo for fruitful discussions and for their invaluable contributes in the design of the multimodal recordings, and the dancers Roberta Messa and Federica Loredan for their kind availability to participate to the recordings of our movement qualities repository.

\section{REFERENCES}

1. Gene Ball and Jack Breese. 2000. Emotion and personality in a conversational agent. Embodied conversational agents (2000), 189-219.

\footnotetext{
${ }^{2}$ H2020 ICT Project DANCE http://dance.dibris.unige.it/
} 
2. Antonio Camurri, Ingrid Lagerlöf, and Gualtiero Volpe. 2003. Recognizing emotion from dance movement: comparison of spectator recognition and automated techniques. International journal of human-computer studies 59, 1 (2003), 213-225.

3. Antonio Camurri, Barbara Mazzarino, Matteo Ricchetti, Renee Timmers, and Gualtiero Volpe. 2004.

Multimodal analysis of expressive gesture in music and dance performances. In Gesture-based communication in human-computer interaction. Springer, 20-39.

4. George Caridakis, Amaryllis Raouzaiou, Elisabetta Bevacqua, Maurizio Mancini, Kostas Karpouzis, Lori Malatesta, and Catherine Pelachaud. 2007. Virtual agent multimodal mimicry of humans. Language Resources and Evaluation 41, 3-4 (2007), 367-388.

5. Georges Dalleau, Alain Belli, Muriel Bourdin, and Jean-René Lacour. 1998. The spring-mass model and the energy cost of treadmill running. European journal of applied physiology and occupational physiology 77 , 3 (1998), 257-263.

6. Paul Fraisse. 1982. Rhythm and tempo. The psychology of music 1 (1982), 149-180.

7. Olivier Girard, Jean-Paul Micallef, and Grégoire P. Millet. 2011. Changes in spring-mass model characteristics during repeated running sprints. European journal of applied physiology 111, 1 (2011), 125-134.

8. Donald Glowinski, Nele Dael, Antonio Camurri, Gualtiero Volpe, Marcello Mortillaro, and Klaus Scherer. 2011. Toward a minimal representation of affective gestures. Affective Computing, IEEE Transactions on 2, 2 (2011), 106-118.
9. Donald Glowinski, Maurizio Mancini, Roddy Cowie, Antonio Camurri, Carlo Chiorri, and Cian Doherty. 2013. The movements made by performers in a skilled quartet: a distinctive pattern, and the function that it serves. Frontiers in Psychology 4, 841 (2013).

10. Chi-Min Hsieh and Annie Luciani. 2005. Generating dance verbs and assisting computer choreography. In Proceedings of the 13th Annual ACM international Conference on Multimedia. ACM, 774-782.

11. Rudolf Laban and Frederick C. Lawrence. 1947. Effort. Macdonald \& Evans.

12. Barbara Mazzarino and Maurizio Mancini. 2009. The Need for Impulsivity \& Smoothness-Improving $\mathrm{HCl}$ by Qualitatively Measuring New High-Level Human Motion Features. In SIGMAP. 62-67.

13. Barbara Mazzarino, Manuel Peinado, Ronan Boulic, Gualtiero Volpe, and Marcelo M. Wanderley. 2009. Improving the Believability of Virtual Characters Using Qualitative Gesture Analysis. In Gesture-Based Human-Computer Interaction and Simulation. Springer, 48-56.

14. John T. McConville, Charles E. Clauser, Thomas D. Churchill, Jaime Cuzzi, and Ints Kaleps. 1980. Anthropometric relationships of body and body segment moments of inertia. Technical Report. DTIC Document.

15. David McNeill. 1992. Hand and mind: What gestures reveal about thought. University of Chicago Press.

16. Pietro Morasso. 1981. Spatial control of arm movements. Experimental brain research 42, 2 (1981), 223-227.

17. Luciana P. Nedel and Daniel Thalmann. 1998. Real time muscle deformations using mass-spring systems. 
In Computer Graphics International, 1998.

Proceedings. IEEE, 156-165.

18. Radoslaw Niewiadomski, Maurizio Mancini, and Stefano Piana. 2013. Human and virtual agent expressive gesture quality analysis and synthesis.

Coverbal Synchrony in Human-Machine Interaction (2013), 269-292.

19. Stefano Piana, Alessandra Stagliano', Antonio Camurri, and Francesca Odone. 2015. Adaptive Body Gesture Representation for Automatic Emotion Recognition. In Transactions on Interactive Intelligent System. ACM press, in printing.

20. Frank E. Pollick. 2004. The features people use to recognize human movement style. In Gesture-based communication in human-computer interaction. Springer, 10-19.

21. Aneesha Singh, Stefano Piana, Davide Pollarolo, Gualtiero Volpe, Giovanna Varni, Ana Tajadura-Jimenez, Amanda CdeC Williams, Antonio Camurri, and Nadia Bianchi-Berthouze. 2016.

Go-with-the-flow: Tracking, Analysis and Sonification of Movement and Breathing to Build Confidence in Activity Despite Chronic Pain. Human-Computer Interaction (2016), 1-49.

22. Paolo Viviani and Tamar Flash. 1995. Minimum-jerk, two-thirds power law, and isochrony: converging approaches to movement planning. Journal of Experimental Psychology: Human Perception and Performance 21, 1 (1995), 32.

23. Harald G. Wallbott and Klaus R. Scherer. 1986. Cues and channels in emotion recognition. Journal of personality and social psychology 51, 4 (1986), 690.
24. Ben R. Whittington and Darryl G. Thelen. 2009. A simple mass-spring model with roller feet can induce the ground reactions observed in human walking. Journal of biomechanical engineering 131, 1 (2009), 011013. 